\title{
Kardiales Lungenödem Ergänzungsprüfung - Das sollten Sie wissen
}

Fallbeispiel Einsatzmeldung „Patient mit Atemnot, Am Stadtgraben 4". Bei der Adresse handelt es sich um eine Altbauwohnung in der Innenstadt - im dritten Obergeschoss. Das Treppenhaus ist extrem eng. Sofort ist dem Team klar: Ein Transport über das Treppenhaus wird hier kaum möglich sein.

Rico Kuhnke • Wolfgang von Meißner

\section{Diagnostik Schritt für Schritt nach ABCDE- und SAMPLER-Schema}

A - Airway Der Patient reagiert sofort auf das Rettungsdienstteam. Aufgrund der massiven Atemnot strengt ihn das Sprechen sehr an. Immer wieder muss er bei der Anam- neseerhebung Sprechpausen einlegen. Aus diesem Grund stellt das Team geschlossene Fragen, auf die der Patient mit Nicken und Kopfschütteln antworten kann. Der Patient ist blass, zyanotisch, und es ist auch ohne Stethoskop ein grobblasiges Rasselgeräusch zu hören (Tipps zur Interpretation: ○ Tab. 1).
B - Breathing Die Atemfrequenz ist mit 30/min stark erhöht. Die Atemzüge sind schwer und das angelegte Pulsoxymeter zeigt eine Sauerstoffsättigung $\left(\mathrm{SpO}_{2}\right)$ von 89\%. Der Patient wird in der sitzenden Position belassen. Zeitgleich erhält er 151 $\mathrm{O}_{2} /$ min über eine Maske mit Reservoir.

C - Circulation Der Puls lässt sich peripher sehr gut tasten, die Frequenz ist mit 120/min tachykard, aber regelmäßig. Der Blutdruck ist mit 110/80 mmHg recht niedrig. Weder die geschilderten Symptome noch ein 12-Kanal-EKG geben Hinweise auf ein akutes Koronarsyndrom (ACS).

Medikamentengabe Der Patient wird nun über die bevorstehenden Maßnahmen 
aufgeklärt. Mit seiner Einwilligung legt das Team einen peripher venösen Zugang. Vor der sublingualen Gabe von Glyzeroltrinitrat-Spray wird der Patient nach Einnahme von potenzsteigernden Medikamenten in den letzten $48 \mathrm{~h}$ befragt und über die mögliche Problematik im Zusammenhang mit der Gabe weiterer Medikamente aufgeklärt. Der Patient verneint die Einnahme, auch eine Medikamentenunverträglichkeit ist ihm nicht bekannt.

- Das Team verabreicht 1 Hub $(0,4 \mathrm{mg})$ Glyzeroltrinitrat-Spray unter die Zunge und prüft den RR engmaschig.

- Weiterhin erhält der Patient 40 mg Furosemid langsam i.v.

\section{Anamnese mittels SAMPLER-Schema} Während sich einer der Kollegen um die Organisation des Transports in die Klinik kümmert und die Feuerwehr als Tragehilfe nachfordert, komplettiert der andere die Anamnese. Hierzu nutzt er das SAMPLERSchema, bevor er die Punkte D und E des anfänglichen ABCDE-Schemas abarbeitet.

- S-Symptome Die Atemnot ist $5 \mathrm{~min}$ nach der Medikamentengabe deutlich besser. Auskultatorisch hört man immer noch ein grobblasiges Rasselgeräusch.

- A-Allergien Allergien sind keine bekannt.

- M-Medikamente Keine regelmäßige Medikamenteneinnahme.

- P - Patientengeschichte Der Patient leidet schon länger unter Kurzatmigkeit. Insbesondere beim Treppensteigen kommt er sehr leicht außer Atem. Heute haben sich die Symptome erstmals nicht gebessert. Beim Hausarzt war der Patient zuletzt vor 1,5 Jahren. Der Arzt hatte ihm dringend angeraten, abzunehmen und den Blutdruck medikamentös einzustellen. Nachdem die erste Tablettenpackung leer war, hat er den Arzt nicht mehr aufgesucht.

- L - Letzte Mahlzeit Vor $1 \mathrm{~h}$ hat der Patient ein Bier getrunken und dazu Chips gegessen.
- E-Ereignis Siehe Patientengeschichte.

- R-Risikofaktoren Der Patient ist 65 Jahre alt, wiegt $108 \mathrm{~kg}$ bei einer Körpergröße von $173 \mathrm{~cm}$ und ist somit stark übergewichtig. Augenscheinlich meidet er Bewegung und ist starker Raucher.

D - Disability Der Patient ist mit einem GCS-Wert von 15 wach und ansprechbar (GCS: Glasgow Coma Scale). Neurologische Defizite sind nicht feststellbar - trotzdem misst das Team noch den Blutzucker. Dieser ist mit $185 \mathrm{mg} / \mathrm{dl}$ deutlich erhöht.

E - Exposure, Environment Bei einer groben körperlichen Inspektion fallen ausgeprägte Beinödeme auf.

Reevaluation nach dem 10-für-10-Prinzip Das Team wiederholt nun nochmals die Punkte A, B und C. Seit Beginn der Versorgung sind $10 \mathrm{~min}$ vergangen.

- Die Atemwege sind weiterhin frei.

- Die Zyanose ist vollständig verschwunden, die $\mathrm{SpO}_{2}$ mit 95\% zufriedenstellend, und die Rasselgeräusche haben deutlich abgenommen.

- Der Blutdruck ist mit $110 / 80$ mmHg stabil, und das Team entscheidet sich für eine Repetition von einem Hub $(0,4 \mathrm{mg})$ Glyzeroltrinitrat-Spray sublingual.

Zustand des Patienten Das Hauptproblem der Atemnot ist unter Kontrolle und die Verdachtsdiagnose „Kardiales Lungenödem bei bestehender Herzinsuffizienz“ am wahrscheinlichsten. Nach wie vor wird der Patient als kritisch angesehen. Einer der Teamkollegen organisiert den Transport, der andere betreut den Patienten.

\section{Tab. 1 Typische Atemgeräusche}

\begin{tabular}{ll} 
Eigenschaft & mögliche Erkrankung \\
\hline feinblasig & typisch für ein Problem in den Alveolen, z. B. bei einer Pneumonie \\
\hline mittelblasig & im Rahmen einer Bronchitis \\
\hline grobblasig & entstehen in Abschnitten mit größerem Lumen, v. a. beim Lungenödem \\
\hline $\begin{array}{l}\text { trockene Atemnebengeräusche } \\
\text { (Pfeifen, Giemen, Brummen) }\end{array}$ & z. B. bei Asthma, chronisch obstruktive Lungenerkrankung (COPD) \\
\hline
\end{tabular}

Eintreffen der Feuerwehr Auch die Kollegen der Feuerwehr sind mittlerweile da - der zuständige Kollege schildert ihnen kurz das Treppenhaus-Problem. Nach einer Begehung gemeinsam mit dem Zugführer der Feuerwehr entscheiden sich die Kollegen für eine Rettung über den Balkon mit der Drehleiter in halbsitzender Position.

Vorbereitung des Patienten Vor der eigentlichen Rettung überlegt das Rettungsteam noch, ob es den Patienten sedieren muss - entscheidet sich aber dagegen. Der Patient wirkt zwar kritisch, aber stabil und ruhig. Schmerzen hat er auch keine. Insofern verzichtet das Team auf eine Gabe von Morphin und Dormicum und klärt den Patienten über den weiteren Rettungsvorgang auf.

\section{Der Algorithmus}

Leitalgorithmen zum Lungenödem Das Rettungsteam im Beispiel nutzt Algorithmen für die Berufsausbildung Notfallsanitäter der Deutschen Angestellten-Akademie (DAA) in Thüringen ( $\triangle$ Abb. 1 und 2). Grundlage für die Algorithmen sind die Musteralgorithmen des Deutschen Berufsverbands Rettungsdienst (DBRD) sowie Leitlinien und Empfehlungen der Fachgesellschaften. Wo keine landeseinheitlichen oder regionalen Algorithmen existieren, sind die Schulen angehalten, diese Lücke für die Ausbildung und Prüfung von Notfallsanitätern zu schließen. Im praktischen Einsatz sind die Ärztlichen Leiter Rettungsdienst bzw. die ärztlichen Verantwortlichen der Rettungsdienste gefordert, sich dieser Aufgabe anzunehmen. 


\section{Leitalgorithmus Lungenödem}

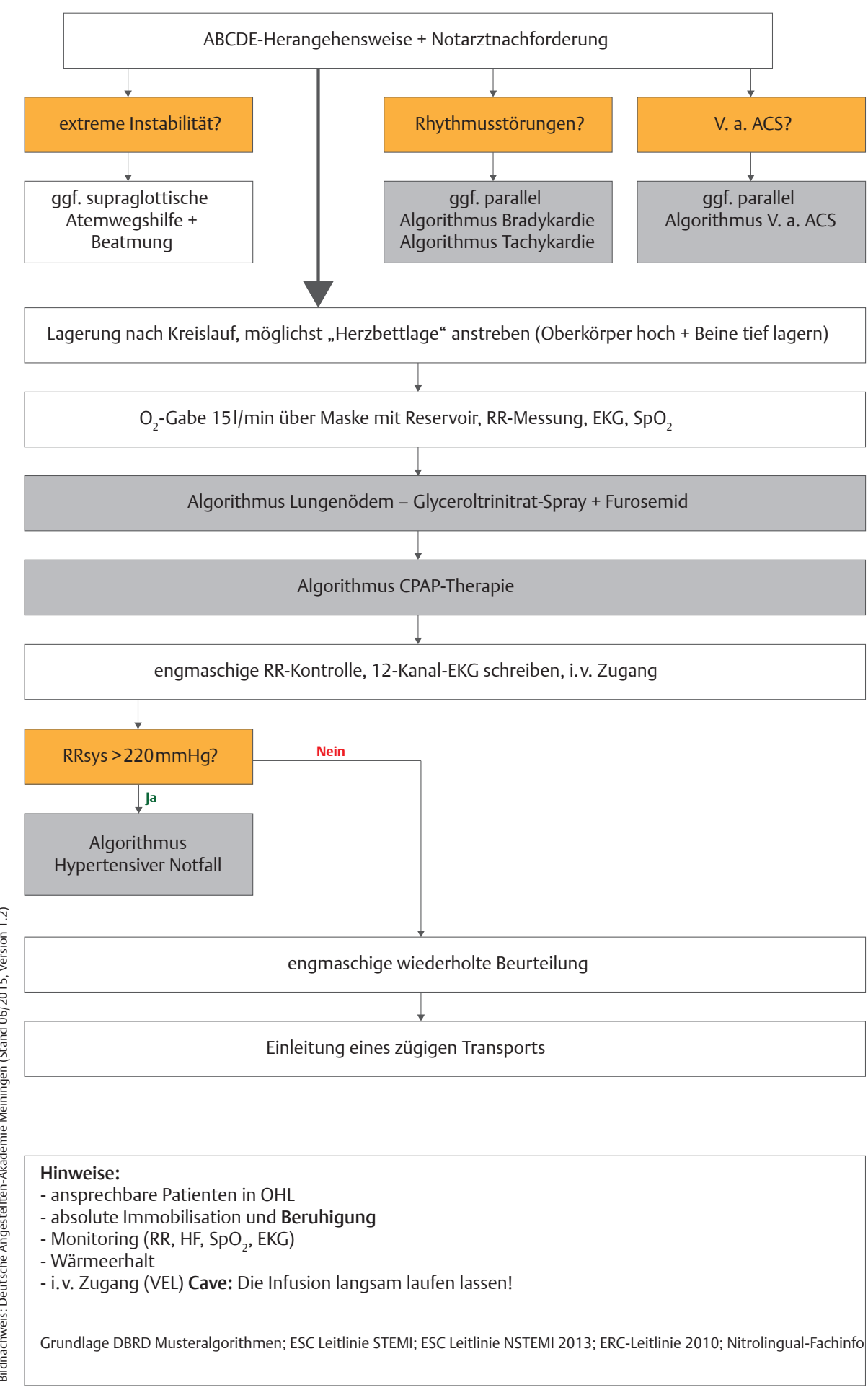

Abb. 1 Vorgehensweise nach dem Leitalgorithmus „Lungenödem “ der Deutschen Angestellten-Akademie Meiningen. ACS: akutes Koronarsyndrom, CPAP: continuous positive airway pressure, HF: Herzfrequenz, OHL: Oberkörperhochlagerung, RR: Blutdruck, $\mathrm{SpO}_{2}$ : Sauerstoffsättigung, VEL: Vollelektrolytlösung.
Sollten Sie in Ihrem Rettungsdienstbereich einen abweichenden Algorithmus nutzen, arbeiten Sie die Unterschiede heraus und überlegen Sie sich, wie der Einsatz mit diesem Algorithmus ablaufen müsste.

Besonderer Rettungsweg Schon beim Eintreffen am Einsatzort wird dem Team die problematische Situation im Treppenhaus bewusst. Die Entscheidung, die Feuerwehr als Tragehilfe anzufordern, ist sinnvoll:

- Der Patient kann weder selbst laufen

- noch liegend im Tragetuch transportiert werden, und

- für den Tragestuhl ist das Treppenhaus zu eng.

Plant das Rettungsdienstteam bereits eine Rettung mit der Drehleiter ein, sollte es diese in jedem Fall mit anfordern.

Diagnosestellung Die Befunde und Anamnese im Beispielfall sprechen für ein kardiales Lungenödem, und das Team kann sich am vorgegebenen Algorithmus entlangarbeiten. Nach der Ersteinschätzung folgt das ABCDE-Schema.

ABCDE-Schema Es besteht eine massive Atemnot, und die auffälligen Rasselgeräusche in Zusammenhang mit der geschilderten Situation lassen auf ein kardiales Lungenödem schließen. Die erhöhte Atemfrequenz und die Sauerstoffsättigung von 89\% unterstreichen die vitale Bedrohung des Patienten. Es besteht weder eine extreme Instabilität, eine extreme Brady- oder Tachykardie noch sind Zeichen für ein ACS festzustellen. Daher kann sich das Team auf den Algorithmus „Lungenödem“ konzentrieren und beginnt die Therapie mit der Lagerung des Patienten sowie der Gabe von Sauerstoff über eine Maske mit Reservoir. 
Herzbettlagerung Hinsichtlich der Lagerung befindet sich der Patient schon in einer optimalen Haltung. Der Sessel im Wohnzimmer hält den Patienten in aufrechter Position und die Beine sind tief gelagert. Hilfreich beim Sitzen kann die Unterstützung durch Kissen im Rückenbereich sein.

Verabreichte Medikamente Die Gabe von Glyzeroltrinitrat-Spray bei einem systolischen Blutdruck von $110 \mathrm{mmHg}$ kann man durchaus kritisch diskutieren. Betrachtet man die pathophysiologischen Probleme des Patienten, ist die Gabe auch bei einem grenzwertigen Blutdruck angebracht. Das Team dosiert hier sehr zurückhaltend mit initial 1 Hub (0,4 mg). Im Anschluss werden die Kreislaufwerte engmaschig überwacht, und es erfolgt eine Repetition bei einem stabilen systolischen Blutdruck von $110 \mathrm{mmHg}$. Zusätzlich werden dem Patienten noch $40 \mathrm{mg}$ Furosemid injiziert.

- Sowohl Glyzeroltrinitrat als auch Furosemid senken die Vorlast am Herzen.

Da der Patient sehr gut auf die Therapie anspricht, kann auf den Algorithmus CPAPTherapie (CPAP: continuous positive airway pressure) verzichtet werden.

Aufgabenverteilung Sehr früh entscheidet sich das Team, die Arbeiten untereinander aufzuteilen. Während ein Kollege die Betreuung des mittlerweile stabilisierten Patienten übernimmt, hat der andere Zeit, Rettung und Transport mit der nachgeforderten Feuerwehr zu organisieren.

Anamnese Bei der Anamneseerhebung nutzt das Team das SAMPLER-Schema. In diesem Fall besonders hilfreich: die Punkte Patientengeschichte und Risikofaktoren.

- Es wird sehr schnell klar, dass der Patient schon seit längerem unter einer Herzinsuffizienz leidet, diese allerdings nicht ärztlich behandelt wird.

- Durch das Weglassen der verordneten Medikamente und das unveränderte

\section{Leitalgorithmus Lungenödem - Glyceroltrinitrat-Spray + Furosemid}

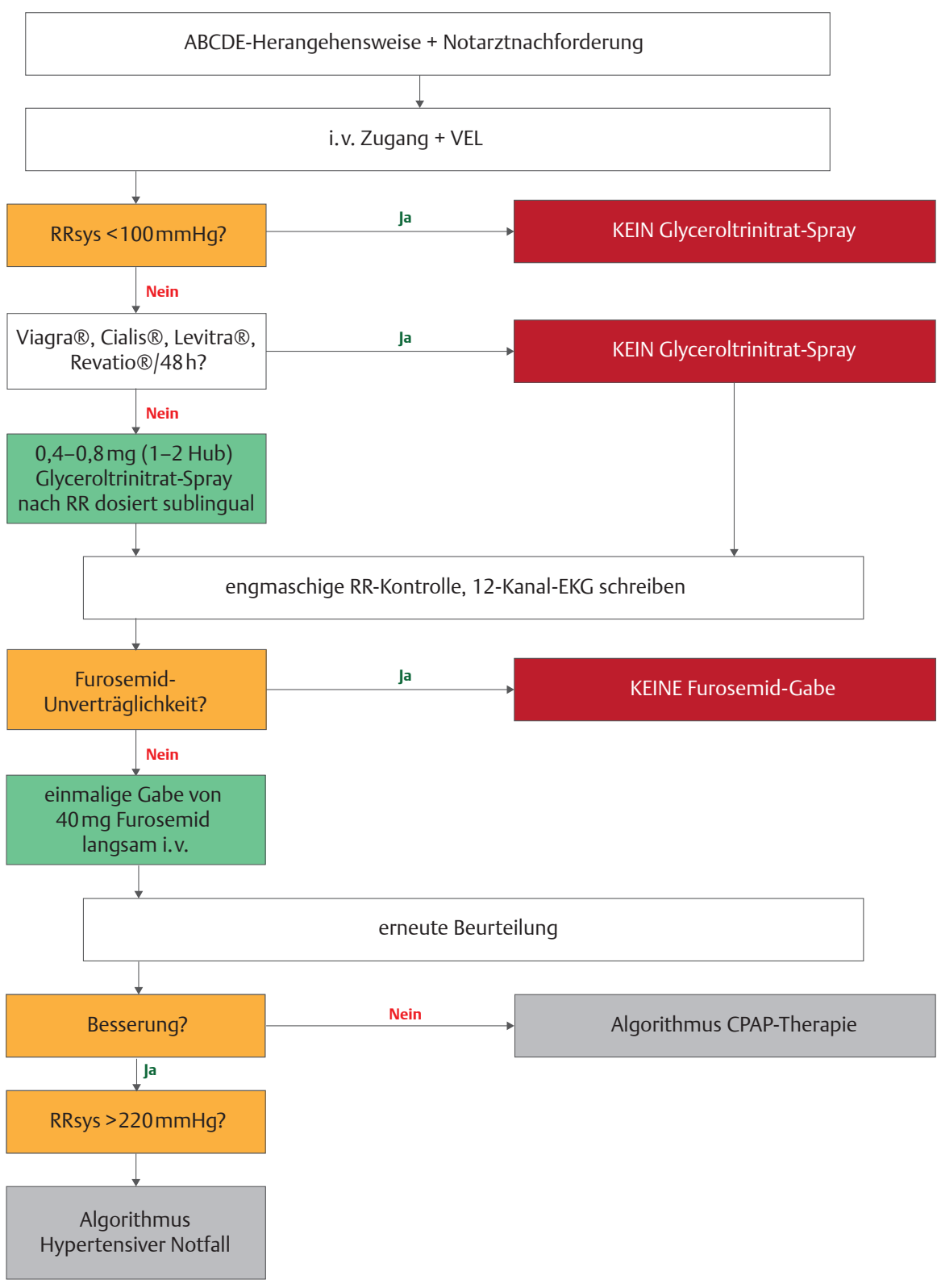

Hinweise:

Grundlage DBRD Musteralgorithmen; ESC Leitlinie Herzinsuffizienz 2012; Nitrolingual-Fachinfo

Abb. 2 Vorgehensweise nach dem Leitalgorithmus „Lungenödem - Glyceroltrinitrat + Furosemid“ der Deutschen Angestellten-Akademie Meiningen. CPAP: continuous positive airway pressure, RR: Blutdruck, VEL: Vollelektrolytlösung.
Verhalten (Adipositas, Rauchen, Bewegungsmangel) ist es zu einer Dekompensation gekommen.
Letzte Schritte Nun erfolgt noch die Klärung von D und $\mathrm{E}$.

- Beinödeme bestätigen den Verdacht auf eine länger bestehende Herzinsuffizienz. 
- Direkt danach erfolgt die Reevaluation der Vitalparameter.

Der Zustand hat sich bis zu diesem Zeitpunkt massiv verbessert, und das Team gibt erneut 1 Hub Glyzeroltrinitrat-Spray bei stabilisierten Kreislaufwerten.

Engmaschige Überwachung Die erneute Beurteilung des Patienten erfolgt nach dem 10-für-10-Prinzip. Das Team wird darin auf aufgefordert, sich in regelmäßigen Abständen (10s für 10 min) kurz Zeit für eine Reevaluation zu nehmen und sich miteinander abzustimmen. Dies geschieht nach folgendem Muster:

1. Hauptproblem benennen

2. Sind alle im Team informiert?

3. Sammeln und Austausch der Fakten

4. nächste Maßnahmen planen

5. Aufgaben im Team verteilen

6. offene Fragen klären

Das 10-für-10-Prinzip ist eine Merkhilfe aus den Leitsätzen des Crisis Resource Managements (CRM) nach Rall \& Gaba [1], die Fehler vermeiden soll.

Jenseits des Algorithmus: Anxiolyse Die Vorbereitung des Patienten für die Rettung via Drehleiter bildet der Algorithmus nicht ab - was die Grenzen von Algorithmen bzw. SOPs verdeutlicht. Das Team im Beispiel erwägt kurzzeitig eine Anxiolyse und Sedierung mittels Morphin und Midazolam. Ohne diese medikamentöse Unterstützung könnte massive Angst bei der ungewöhnlichen Rettung zur erneuten Dekompen-

\section{Welche Algorithmen nutzen Sie?}

Um die Serie für unsere Leser möglichst attraktiv und ansprechend zu gestalten, verwenden wir die unterschiedlichsten Algorithmen und SOPs aus dem gesamten Bundesgebiet. Wir würden uns freuen, wenn Sie uns hierbei unterstützen. Senden Sie uns Ihre regionalen Algorithmen und SOPs zusammen mit Ihren Kontaktdaten an: retten@thieme.de. sation führen. Allerdings ist der Patient schmerzfrei und stabil. Es besteht zudem keine Atemnot, und die kardiale Vorlast wurde ausreichend gesenkt. Midazolam birgt in dieser Situation die Gefahr der Atemdepression - auch, weil der Patient als starker Raucher unter einer chronisch obstruktiven Lungenerkrankung (COPD) leiden könnte! Das Team handelt richtig, wenn es auf die medikamentöse Sedierung verzichtet. Ansonsten hätte es dafür den Notarzt nachfordern müssen.

\section{NOTFALLMEDIZIN}

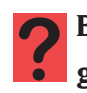

Beschreiben Sie die Pathophysiologie eines kardialen Lungenödems und erläutern Sie in dem Zusammenhang die Wirkungsweise von Sauerstoffgabe, Glyzeroltrinitrat und Furosemid.

Flüssigkeitsstau durch Herzinsuffi-

zienz Ursache für ein kardiales Lungenödem ist eine akute Linksherzinsuffizienz, in deren Folge es zu einem Rückstau des Blutes in die Gefäße des Lungenkreislaufs kommt. Der Rückstau erhöht den Druck im Gefäßsystem - Flüssigkeit wird aus den Kapillaren über den Zellzwischenraum in die Alveolen gepresst.

- Die Flüssigkeitsansammlung in den Alveolen vergrößert die Diffusionsstrecke für den Sauerstoff, sodass dieser schlechter diffundieren kann.

- So nimmt der zirkulierende Sauerstoff drastisch ab und damit auch die $\mathrm{SpO}_{2}$.

Wirkmechanismus der Sauerstoffgabe Die hochdosierte Gabe von Sauerstoff über eine Maske mit Reservoir erhöht den Sauerstoffgehalt in der Einatemluft auf über $90 \%$. Durch den größeren Diffusionsgradienten kann Sauerstoff leichter durch die Kapillaren in das Blutgefäßsystem diffundieren als beim normalen Sauerstoffgehalt von $21 \%$ in der Umgebungsluft.
Effekt von Nitroglyzerin Glyzeroltrinitrat wirkt auf die Koronargefäße am Herzen wie auch auf die periphervenösen Gefäße erweiternd. Diese Gefäßerweiterung (sog. venöses Pooling) senkt die Vorlast - das Herz wird deutlich entlastet.

- Das weniger belastete Herz ist wieder besser in der Lage, das ankommende Blut aus dem rechten Herzen in den Körperkreislauf zu pumpen.

- In der Folge sinkt auch der Druck im Lungenkreislauf, was dem Lungenödem entgegenwirkt.

Eigenschaften von Furosemid Auch Furosemid bewirkt ein periphervenöses Pooling und hilft die Vorlast zu senken. Nach ca. 1020 min tritt außerdem sein Effekt als sog. Schleifendiuretikum ein: Es reduziert die Rückresorption von Wasser in den Nieren und führt zu einer verstärkten Harnbildung. Mit der Reduktion des zirkulierenden Volumens strebt man ebenfalls eine Entlastung des Herzens an.

Nach der Gabe von Furosemid ist es unbedingt erforderlich, den Patienten über den verstärkten Harndrang zu informieren und ihm die Möglichkeit zum Wasserlassen zu geben.

Mögliche weitere Fragen:

2 Welche anderen Ursachen für ein Lungenödem kennen Sie? Erläutern Sie die zugrundeliegende Pathophysiologie.

Beurteilen Sie den Einsatz von nicht invasiver bzw. CPAP-Beatmung und erläutern Sie Ihre Vorgehensweise. Was versteht man unter Diffusion? Beschreiben Sie Faktoren, die diese begünstigen oder erschweren. 


\section{KOMMUNIKATION}

?

Sie möchten Ihren Patienten auf die

Rettung mithilfe der Drehleiter vorbereiten und sagen: „Leider ist ein Transport durchs Treppenhaus nicht möglich und wir müssen Sie mit einer Drehleiter über den Balkon retten.“ Wie kann der Patient diesen Satz verstehen? Benutzen Sie für Ihre Argumentation das Kommunikationsmodell von Schulz von Thun.

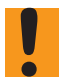

Vier-Ohren-Modell Der Patient kann den Satz auf unterschiedliche Weise interpretieren. Der Empfänger einer Information entscheidet über den Inhalt. Daher ist es wichtig, sich zu vergewissern, ob die Information auch so ankommt, wie man sie gemeint hat. Schulz von Thun unterscheidet in seinem Vier-Ohren-Modell 4 Interpretationsmöglichkeiten einer Information:

Sachebene Auf der Sachebene geht es darum, wie der eigentliche Sachverhalt zu verstehen ist. Hier lautet die Information:

- „Ein Transport über das Treppenhaus ist nicht möglich. Sie werden über den Balkon mit einer Drehleiter transportiert."

Selbstoffenbarungsebene Auf der Ebene der Selbstoffenbarung gibt der Sender einen Blick in das eigene Empfinden preis. Im Beispiel könnte dies bedeuten:

- „Leider sind wir nicht in der Lage, Sie durch das Treppenhaus zu tragen und müssen Ihnen den Transport mit der Drehleiter zumuten.“

Beziehungsebene Die Beziehungsebene spiegelt wider, wie sich der Empfänger vom Sender verstanden und wertgeschätzt fühlt. Im Beispiel könnte der Empfänger Folgendes interpretieren:

- „Der findet mich zu fett und ärgert sich, weil ich ihm Probleme mit meinem Transport zumute.“
- Oder aber: „Der ist sehr bemüht und möchte mir die bestmögliche Versorgung zukommen lassen.“

Apellebene Auf der Ebene des Apells stellt sich der Empfänger die Frage, was die Information für eine Zielsetzung hat. Dies könnte sein:

- „Stellen Sie sich nicht so an und laufen Sie nach unten." oder

- „Machen Sie sich keine Sorgen, wir haben alles im Griff.“

Das Modell von Schulz von Thun macht v. a. deutlich, wie unterschiedlich der Empfänger eine Information interpretieren kann. Für den Rettungsdienst bedeutet dies:

$\triangleright$ Vergewissern, ob die Information auch wie gewünscht beim Empfänger angekommen ist und

$\triangleright$ viel Wert darauf legen, in welchem Ton man mit dem Patienten spricht.

Mögliche weitere Fragen:

$?$ Welche Ängste könnte der Patient hinsichtlich der Rettung mit der Drehleiter haben und wie gehen Sie damit um?

$?$ Wie gehen Sie damit um, wenn Ihr Patient die Einwilligung in die invasiven Maßnahmen verweigert? Bei der Diskussion über die geeignete Methode zur Rettung des Patienten geraten Sie mit dem Zugführer in Streit. Beschreiben Sie Möglichkeiten, hier deeskalierend zu wirken.

\section{RAHIMENBEDINGUNGEN}

Wie beurteilen Sie die Entscheidung, die Feuerwehr zur Hilfe nachzufordern? Halten Sie die Rettung mit der Drehleiter für sinnvoll und welche Alternativen könnten Sie nutzen?
Bei Bedarf Unterstützung nachfordern Typischerweise arbeitet ein Team im Notfalleinsatz mit 2 (RettAss/ NotSan + RettSan) bis 5 Personen (dann zusätzlich noch NEF-Fahrer, Notarzt und ggf. Praktikant auf dem RTW). Je nach Situation kann es trotzdem erforderlich sein, zusätzliche Hilfe anzufordern.

- Insbesondere bei engen Treppenhäusern kann sich der Transport als überaus schwierig bis unmöglich gestalten.

- Daher macht es Sinn, zusätzliche Personen zur Unterstützung nachzufordern.

Einsatz der Drehleiter Bei der Entscheidung über die geeignete Art der Rettung steht der Patientenzustand im Vordergrund. Im Beispiel ist das Treppenhaus sehr eng und ein Transport über die Treppe würde den Patienten stark belasten. Im Idealfall transportiert man den Patienten sitzend.

- Der Transport mit der Drehleiter scheint in diesem Fall der beste zu sein.

Vor- und Nachteile anderer Möglichkeiten Alternativ bietet sich die Rettung mit einem Tragestuhl oder einem Tragetuch an.

- Vonseiten der Lagerung und Belastung des Patienten wäre der Transport mit dem Tragestuhl sicherlich die schonendste Alternative. Limitierender Faktor ist hier die Größe des Treppenhauses.

- Der Transport mit dem Tragetuch benötigt hier deutlich weniger Raum - allerdings ist dann ein sitzender Transport nur sehr eingeschränkt möglich.

Eine Entscheidung muss im konkreten Einsatz unter Berücksichtigung aller Möglichkeiten gemeinsam mit allen Beteiligten geschehen.

\section{Mögliche weitere Fragen:}

Welche Anforderungen an die Dokumentation müssen Sie insbesondere bei der Anwendung von erweiterten Maßnahmen erfüllen? 


\section{Welche Belastungsgrenzen hinsicht- lich des Körpergewichts Ihrer Pati- enten bestehen für Ihre Fahrtrage, Schaufeltrage und Ihr Tragetuch? Beschreiben Sie die besonderen Anforderungen bei Rettung und Transport stark adipöser Personen.}

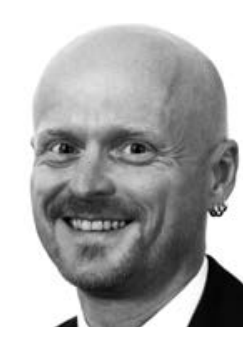

Rico Kuhnke ist Schulleiter der DRK-Landesschule Baden-Württemberg. Er war viele Jahre als Lehrrettungsassistent tätig und ist Notfallsanitäter. Er ist Mitherausgeber von retten!.

E-Mail:

r.kuhnke@drk-ls.de

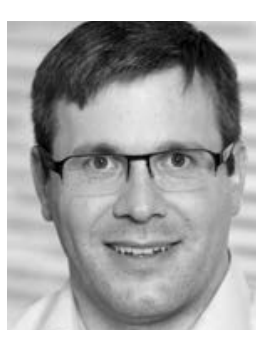

Dr. Wolfgang von Meißner, MHBA, ist Ärztlicher Leiter der DRK-Landesschule Baden-Württemberg und ERC-ALS Course Director. Als Facharzt für Anästhesiologie mit den Zusatzbezeichnungen Notfallmedizin und Intensivmedizin absolviert er die Weiterbildung zum Facharzt für Allgemeinmedizin im Quereinstieg. Er ist Mitherausgeber von retten!.

E-Mail: vonmeissner@hausaerzte-am-spritzenhaus.de

\section{Literatur}

1 Rall M, Gaba DM. Human performance and patient safety. In: Miller RD, Hrsg. Miller's Anesthesia. Philadelphia: Elsevier Churchhill Livingstone; 2009: $93-150$

\section{Kommentar von Sebastian Koch, SRH Fachhochschule für Gesundheit Gera}

Am 7. Juli 2015 hat die Prüfungskommission des Landesverwaltungsamts Thüringen ihren Lehrplan für Notfallsanitäter präsentiert. Erarbeitet wurde er von der Lehrplankommission - bestehend aus 6 ausgewählten Lehrern staatlich anerkannter Thüringer Schulen, die Rettungsassistenten ausbilden. Leitung und Zusammenstellung oblagen Carmen Frey (Referentin des Thüringer Instituts für Lehrerfortbildung, Lehrplanentwicklung und Medien - ThILLM). Des Weiteren haben Institutionen, die an der Notfallsanitäter-Ausbildung beteiligt sind, das Gremium als externe Fachberater unterstützt - so z. B. Rettungswachen, Krankenhäuser, Landesärztekammer und die Arbeitsgemeinschaft Thüringer Notärzte. Das Grundprinzip der NotSan-APrV - erlerntes theoretisches Wissen zeitnah während der Praktika zu vertiefen - war von Beginn an bei der Erstellung des Lehrplans bestimmend. Ziel war es, für alle Rettungsdienstbereiche in Thüringen einheitliche Handlungsalgorithmen auf Grundlage des aktuellen Stands von Wissenschaft und Technik zu entwickeln und in die Ausbildung zu integrieren.

Die im Thüringer Lehrplan für Notfallsanitäter festgelegten erweiterten notfallmedizinischen Kompetenzen orientieren sich am Pyramidenprozess, der durch den Bundesverband Ärztlicher Leiter Rettungsdienst initiiert wurde. Alle enthaltenen Maßnahmen sind durch den Landesverband der Ärztlichen Leiter Rettungsdienst (ÄLRD) Thüringen sowie der Arbeitsgemeinschaft Thüringer Notärzte bestätigt - daher können die zukünftigen Notfallsanitäter diese selbstständig und eigenverantwortlich anwenden.

Auf Grundlage der „Musteralgorithmen zur Umsetzung des Pyramidenprozesses im Rahmen des NotSanG “ (veröffentlicht vom Deutschen Berufsverband Rettungsdienst) sowie aktueller wissenschaftlicher Erkenntnisse der jeweiligen Fachgesellschaften und Institutionen, hat die Deutsche Angestellten-Akademie Meiningen (DAA) einen Algorithmenkatalog für die Berufsausbildung Notfallsanitäter erstellt - unter intensiver Beteiligung des ÄLRD Schmalkalden/Meiningen, Dr. Michael Walther.

Dieser Katalog beinhaltet derzeit (Stand: 28.11.2015) 55 Handlungsalgorithmen unterschiedlicher Notfallbilder. Er erhebt keinen Anspruch auf Vollständigkeit und wird auch künftig mit kritisch-konstruktivem Blick betrachtet und fortgeschrieben. Er steht für eine innovative und qualitative Gestaltung der Ausbildung. Auf Grundlage des aktuellen Stands von Wissenschaft und Technik entwickelt die DAA die Algorithmen stetig weiter. Der Landesverband der ÄLRD Thüringen erarbeitet zudem grundsätzliche Kernalgorithmen, um in allen Rettungsdienstbereichen Thüringens einen einheitlichen Algorithmenkatalog bereitzustellen - und damit ein landesweit einheitliches Vorgehen bei invasiven bzw. erweiterten Versorgungsmaßnahmen durch Notfallsanitäter zu erreichen. Handlungsalgorithmen geben dem Notfallsanitäter Handlungssicherheit, dem Patienten die bestmögliche standardisierte Versorgung und Ärztlichen Leitern / Notärzten Transparenz über das Handlungsspektrum des neuen Berufsbilds. Zudem ermöglicht es gemäß § 4 Abs. 2 Punkt C dem ÄLRD, die invasiven Maßnahmen festzulegen, zu überprüfen und schlussendlich zu verantworten.

Sebastian Koch ist Dozent der Deutschen Angestellten-Akademie Meiningen und wissenschaftlicher Mitarbeiter im Studiengang Medizinpädagogik an der SRH Fachhochschule für Gesundheit Gera. E-Mail: sebastian.koch@srh-gesundheitshochschule.de

Beitrag online zu finden unter http://dx.doi.org/10.1055/s-0041-107472 